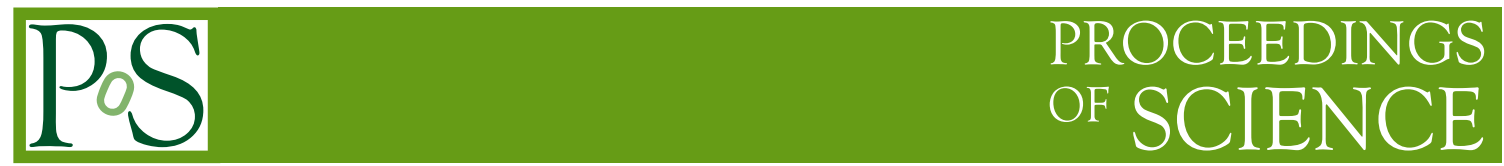

\title{
CMS ECAL monitoring and calibration in LHC Run 2
}

\author{
Fabio Monti* on behalf of the CMS collaboration \\ University and INFN of Milano-Bicocca, Italy \\ E-mail: fabio.monti@cern.ch
}

\begin{abstract}
Precise calibration and monitoring of the CMS electromagnetic calorimeter (ECAL) is a key ingredient in achieving the excellent ECAL performance required by many physics analyses employing electrons and photons. This poster describes the methods used to monitor and intercalibrate the ECAL response, using physics channels such as W/Z boson decays to electrons and pi0/eta decays to photon pairs, and also exploiting the azimuthal symmetry of the minimum bias events. Results of the calibrations obtained with Run 2 data are presented.
\end{abstract}

Sixth Annual Conference on Large Hadron Collider Physics (LHCP2018)

4-9 June 2018

Bologna, Italy

${ }^{*}$ Speaker. 


\section{The CMS ECAL}

The CMS electromagnetic calorimeter (ECAL) is a hermetic homogeneous calorimeter made up of 75848 scintillating lead tungstate crystals $\left(\mathrm{PbWO}_{4}\right), 61200$ placed in a barrel, the remaining ones (14648) in two endcaps, as shown in Figure 1. For photons and electrons with energy larger than about $10 \mathrm{GeV}$, one of the main contributions to the energy resolution comes from the singlechannel response uniformity and stability. Therefore the ECAL needs precise inter-calibration and energy scale monitoring systems.

Luminosity of Run 2 is about $10-20 \cdot 10^{33} \mathrm{~cm}^{-2} \mathrm{~s}^{-1}$, compared to $6-7 \cdot 10^{33} \mathrm{~cm}^{-2} \mathrm{~s}^{-1}$ of Run 1 , with about the same duration of data taking. Larger luminosity ensures a larger dataset for the analysis, but also implies a larger radiation damage on ECAL crystals that needs to be effectively monitored.

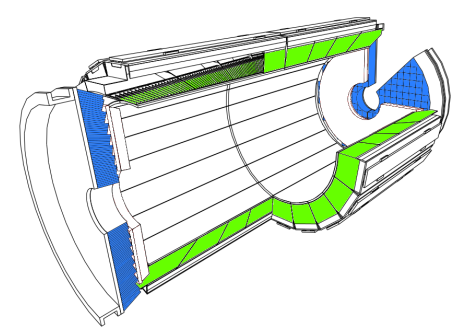

Figure 1: Schematic ECAL layout. In green the barrel and in blue the endcaps. The longitudinal length and the diameter of the ECAL are respectively about $6 \mathrm{~m}$ and $2.6 \mathrm{~m}$.

\subsection{The role of the ECAL in CMS Run 2}

The ECAL plays a fundamental role in many physics analyses employing electrons and photons such as precision measurements of the Higgs boson, and searches for new phenomena beyond the standard model.

Interesting observables to characterize the Higgs boson include the mass, the fiducial and differential cross sections, and the couplings. They have to be precisely measured in order to make a precision test of the standard model. For this purpose, the ECAL performance is fundamental for the decay channels $H \rightarrow \gamma \gamma, H \rightarrow Z Z \rightarrow e e \mu \mu$, and $H \rightarrow Z Z \rightarrow 4 e$. The ECAL is fundamental also in the search for high energy resonances (TeV scale) decaying in diphoton or dielectron predicted by some theories beyond the standard model, as for example the $Z^{\prime}$ particle decay in dielectron [1].

\section{Monitoring and correction for ECAL response changes}

The ECAL response changes over time mainly because of crystal transparency variations due to radiation damage and recovery [2]. The change of crystal transparency depends on the absorbed dose, therefore on the time and pseudorapidity $(\eta)$. Moreover, during periods without collisions, the radiation damage is partially recovered. A snapshot of the transparency history of the ECAL crystals is shown in Figure 2a for different $\eta$ regions. In order to measure and correct for crystal transparency changes, blue and green laser lights (respectively $440 \mathrm{~nm}$ and $495 \mathrm{~nm}$ ) are injected during a fraction of the beam abort gaps [3]. A scan of whole ECAL with one laser takes about $20 \mathrm{~min}$, hence $40 \mathrm{~min}$ for blue and green laser scans. 
Within 48 hours from data-taking a prompt-processing of data is performed for the so-called "prompt-reco". Response corrections based on laser data must be provided within this time interval. After the prompt-reco, ECAL stability over time is validated using physics events from collisions.

\subsection{Validation of ECAL stability with $\pi^{0} \rightarrow \gamma \gamma$ events}

The invariant mass peak in $\pi^{0} \rightarrow \gamma \gamma$ events is monitored over time as shown in Figure 2b. Events for this task comes from a special stream of data from a dedicated trigger. Events are divided into time bins. For each bin, the $M_{\gamma \gamma}$ distribution is fitted with a proper function for signal and background. The photons from $\pi^{0}$ decay are at the low limit of the ECAL energy range of interest, their relatively low energy makes this method slightly sensitive to pileup, which is not constant during a LHC fill.

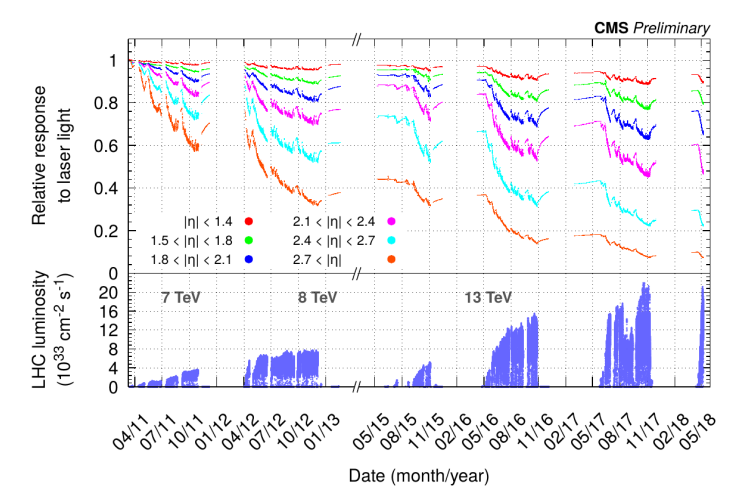

(a)

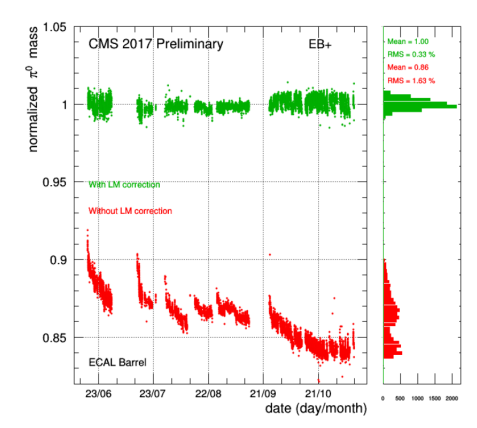

(b)

Figure 2: (a): on the upper plot ECAL response over time at different $\eta$ regions as monitored by laser since 2011. On the lower plot the corresponding value of luminosity. (b): ECAL response over time monitored with the invariant mass peak in $\pi^{0} \rightarrow \gamma \gamma$ events. Red points represent data not corrected for laser data, while green points represent the scale corrected for that.

\subsection{Validation of ECAL stability with $W \rightarrow e v$ events}

Further monitoring of the ECAL stability is provided by the ratio $E / p$ of electrons from $W \rightarrow e v$ decay, where $E$ is the energy measured by ECAL and $p$ is the momentum measured by the tracker, taken as reference. For a stable and calibrated detector, the $E / p$ distribution is expected to be centered at 1 . Events are divided in time intervals with about the same amount of data. For each interval, the $E / p$ distribution is fitted with a template distribution derived from the data (Figure 3a). The E/p scale parameter is extracted from the fit and its value is monitored over time, as shown in Figure 3b. The time granularity of this method is about 1 point every 10-20 min, limited by the cross section and the trigger efficiency.

\subsection{Validation of ECAL stability with $Z \rightarrow e e$ events}

The invariant mass peak in $Z \rightarrow e e$ events is monitored over time as shown in Figure 4. Events are divided into time intervals with about the same number of events. For each time interval the 


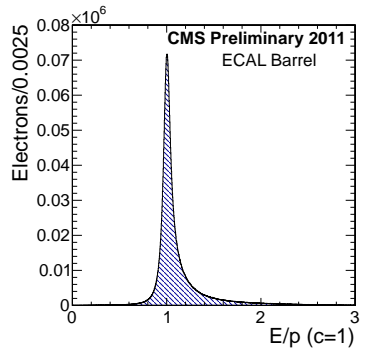

(a)

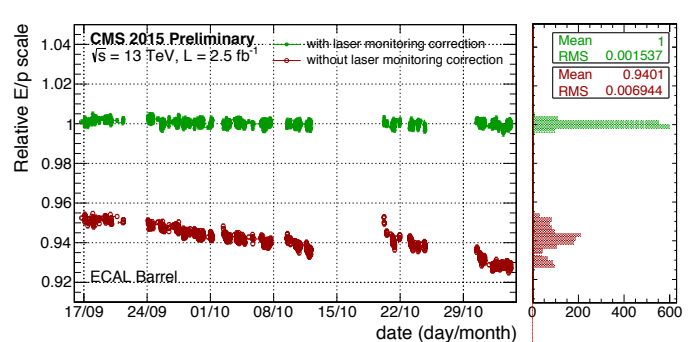

(b)

Figure 3: (a): $E / p$ distribution. (b): ECAL response over time as monitored using $E / p$ peak position for electrons from $W \rightarrow e v$ events. Red points represent data not corrected for crystal transparency changes, while green points represent the scale corrected for that.

median of $M_{e e}$ distribution is computed. Values of the measured median, normalized to $Z$ mass [4], are monitored over time, as shown in Figure 4. The rate of $Z \rightarrow e e$ events, including the trigger efficiency, is lower than the rate of $W \rightarrow e v$ events, in particular the achievable time granularity is about 1 point every 1-2 hours. Electrons coming from $Z$ decays, as well as the ones from $W$ decay, have energy of the order of about $40-50 \mathrm{GeV}$. Therefore, the ECAL response stability is monitored exactly in the range of interest for physics analysis.

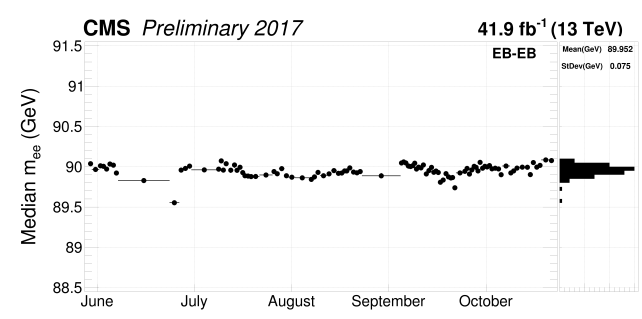

Figure 4: ECAL response over time monitored using the invariant mass median in $Z \rightarrow e e$ events.

\section{Intercalibration in Run 2}

The purpose of the ECAL crystal intercalibration is to improve the energy resolution reducing the channel-to-channel response spread. The intercalibration constants are computed typically at the end of each year of data taking profiting of the full amount of data. A subsequent reconstruction of the data including intercalibration constants is performed. Four methods have been developed to intercalibrate crystals. Basically the energy scale parameters are the same ones used for the monitoring, therefore $E / p$ ratio for electrons from $W \rightarrow e v$, invariant mass peak in $\pi^{0} \rightarrow \gamma \gamma$ events, and invariant mass peak in $Z \rightarrow e^{+} e^{-}$events. In addition, the $\phi$-symmetry method uses the average energy deposited in each crystal of a given barrel $\eta$-ring for minimum bias events, exploiting the symmetry along $\phi$. Intercalibration constants computed with the different methods are combined together and the resulting IC are applied to data. The residual miscalibration in 2017, shown in Figure 5, is between 0.2 and $1 \%$, depending on $\eta$, which corresponds to about $1 \%$ and $1-2 \%$ improvement in the resolution of the barrel (Figure 6a) and the endcaps (Figure 6b), respectively. 
The improvements are estimated from the width of the invariant mass distribution of $Z \rightarrow e e$ events.

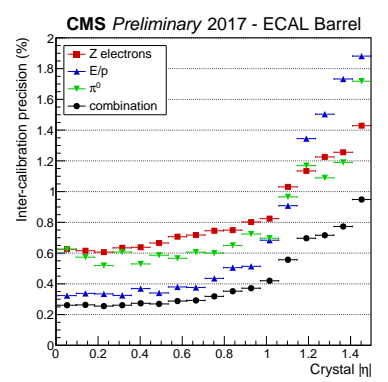

Figure 5: Intercalibration precision computed from the IC combination as a function of $\eta$.

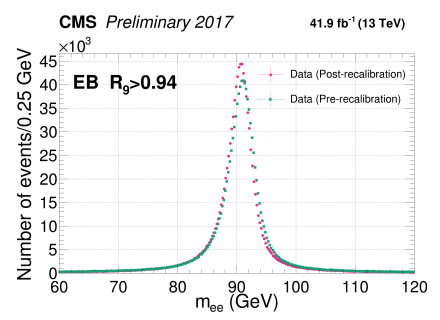

(a)

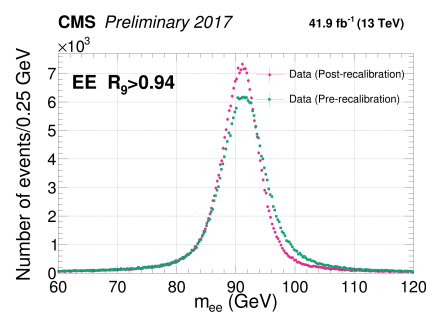

(b)

Figure 6: Dielectron invariant mass for $Z \rightarrow e e$ events before (green) and after (pink) intercalibration for the barrel (a), and for the endcaps (b).

\section{References}

[1] CMS collaboration, A. M. Sirunyan et al., Search for high-mass resonances in dilepton final states in proton-proton collisions at $\sqrt{s}=13 \mathrm{TeV}$, JHEP 06 (2018) 120 [1803.06292].

[2] T. Adams, P. Adzic, S. Ahuja, D. Anderson, M. Andrews, I. Antropov et al., Beam test evaluation of electromagnetic calorimeter modules made from proton-damaged pbwo 4 crystals, Journal of Instrumentation 11 (2016) P04012.

[3] M. Anfreville, D. Bailleux, J. Bard, A. Bornheim, C. Bouchand, E. Bougamont et al., Laser monitoring system for the cms lead tungstate crystal calorimeter, Nucl. Inst. and Methods in Physics Research Section A: Accelerators, Spectrometers, Detectors and Associated Equipment 594 (2008) 292.

[4] Particle Data Group collaboration, K. A. Olive et al., Review of Particle Physics, Chin. Phys. C38 (2014) 090001.

[5] CMS collaboration, The CMS electromagnetic calorimeter project: Technical Design Report, Technical Design Report CMS. CERN, Geneva, 1997.

[6] CMS collaboration, CMS ECAL with 2017 data, CMS Detector Performance Summaries (2018) .

[7] CMS collaboration, CMS ECAL Laser monitoring up to $2017, \pi^{0} / \eta \rightarrow \gamma \gamma$ spectrum and monitoring, ES calibration, CMS Detector Performance Summaries (2017) .

[8] CMS collaboration, ECAL Laser monitoring till end of 2016 and ECAL phi-symmetry, CMS Detector Performance Summaries (2017). 